\title{
FORMAÇÃO PROFISSIONAL EM EDUCAÇÃO FÍSICA: ESTADO DA ARTE 1
}

\author{
Natalia Papacídero Magrin \\ Universidade Federal Do Triângulo Mineiro \\ natimagrin@hotmail.com \\ Regina Maria Rovigati Simões \\ Universidade Federal Do Triângulo Mineiro \\ rovigatisimoes@uol.com.br \\ Wagner Wey Moreira \\ Universidade Federal Do Triângulo Mineiro \\ weymoreira@uol.com.br
}

RESUMO: O objetivo deste estudo é analisar como está a produção literária na área da Educação Física (EF) e Esportes após a separação legal entre Licenciatura e Bacharelado. Metodologia: Estudo de revisão, do tipo estado da arte, o qual analisou qualitativamente a produção literária em EF. Resultados: Foram encontrados 28 artigos e divididos por assunto, estabelecendo-se três categorias: 1) Formação/Graduação, 16 artigos, 2) Atuação Profissional, 10 artigos, 3) Área de Conhecimento, 2 artigos. Conclusão: Ficou evidente um conflito entre os cursos de Formação Profissional em EF, não sendo possível verificar um padrão de formação para as diferentes habilitações da área.

Palavras Chave: Educação Física. Esportes. 


\section{Introdução}

Na última década muitos autores se dedicaram a definir a Educação Física (EF) enquanto "profissão", na tentativa de utilizar o termo sem as aspas que tem Ihe acompanhado há algum tempo, por acreditarem ser um termo legítimo e empregado por muitos de maneira errônea. Neste sentido, alguns escritos têm surgido: uns para diferenciar os cursos de licenciatura dos cursos de bacharelado e ainda justificar a necessidade ou não desta divisão; outros preocupados com suas consolidações legais, ou então, buscando entender a Educação Física enquanto área de conhecimento, demarcando seus instrumentos de estudo.

As discussões sobre a formação profissional em Educação Física ganharam proporções principalmente após o ano de 2000, fruto da consolidação do Conselho de Educação Física, do aumento do número de cursos de graduação e de pós-graduação, bem como da produção de conhecimento na área.

Ao acompanhar as reformas educacionais no Brasil, na década de 90 do século XX, nota-se que a EF adentrou nas discussões sobre a formação profissional específica para professores da Educação Básica, da mesma forma que buscou ampliar a formação para as demais áreas de atuação que estavam em emergência, dentre elas lazer, treinamento físico (clubes, academias), trato para com pessoas portadoras de necessidades especiais, esporte de aventura, dentre outras. Discussões e reflexões a respeito da formação profissional em EF, nas vertentes bacharelado e ou licenciatura ainda estão em sua gênese, mas já encontramos vários profissionais se debruçando sobre o assunto. (DARIDO, SILVA, 2002; TEREZANI, 1994; RANGEL-BETTI, BETTI, 1996).

Frente aos conflitos, o Ministério da Educação do Brasil junto à Secretaria de Educação Superior, lançou no ano de 2010, os Referenciais Curriculares Nacionais dos Cursos de Bacharelado e Licenciatura (BRASIL, 2010). Neste, os cursos foram contemplados com algumas informações para a elaboração dos futuros currículos: carga horária mínima, tempo de integralização, perfil do egresso, temas abordados na formação, ambientes de atuação e infraestrutura recomendada.

Observa-se nesta trajetória um movimento de pesquisas envolvendo a formação de professores, a estruturação de currículos, a profissão docente, as metodologias de ensino, as políticas de formação, entre outros. Estas pesquisas chegam à comunidade acadêmica 
na forma de artigos científicos, monografias, dissertações e teses, objetivando discutir e aprimorar a profissão em todos seus âmbitos de formação.

$\mathrm{Na}$ ânsia de conhecer a totalidade de estudos e pesquisas a cerca de um fenômeno, muitos autores tem se dedicado as produções denominadas de "Estado da Arte" as quais, segundo Ferreira (2002), passam por dois momentos. Um primeiro em que se "interage com a produção acadêmica através da quantificação e de identificação de dados bibliográficos, com o objetivo de mapear essa produção num período delimitado" (FERREIRA, 2002, p. 265). Um segundo momento, quando o pesquisador "deve buscar responder, além das perguntas "quando", "onde" e "quem" produz num determinado período e lugar, àquelas questões que se referem a "o quê" e "o como" dos trabalhos" (FERREIRA, 2002, p. 265).

Este é um estudo de revisão literária, do tipo estado da arte, o qual analisou qualitativamente a produção literária em Educação Física a partir dos seguintes critérios: (a) Publicações nacionais disponíveis no Portal de Periódicos CAPES; (b) produções do período de 15/10/2005 à 18/05/2014, (c) inclusão no título e/ou assunto das publicações, os seguintes descritores: 1. Educação Física e Formação Profissional; 2. Educação Física e Bacharelado; 3. Educação Física e Licenciatura.

O período das publicações escolhido justifica-se devido à nota técnica a qual estabelece:

[...] o curso de Educação Física pôde ser ofertado nas modalidades bacharelado e licenciatura plena conjuntamente, de forma regular, até findo o prazo máximo para adequação das Diretrizes Nacionais de Curso (DCNs) determinado pelo Conselho Nacional de Educação (CNE), ou seja, até 15/10/2005 [...] após essa data as modalidades do curso de Educação Física passaram a representar graduação distinta. (BRASIL, 2013, p.4)

Vale ressaltar que os trabalhos repetidos foram descartados e não houve critério de seleção quanto ao tipo de publicação, se eram teses, dissertações ou artigos, porem ocorreu que $100 \%$ das publicações encontradas foram em formato de artigos.

Neste sentido o objetivo deste estudo é analisar o estado da arte das produções literárias em Educação Física, no que diz respeito à formação em graduação, após a separação legal entre Licenciatura e Bacharelado. 


\section{Resultados e Discussão}

Fazem parte da produção literária em Educação Física e Esportes, de acordo com os critérios preestabelecidos, 28 artigos. Destes, 25 foram encontrados com os descritores Educação Física e Formação Profissional, 2 com Educação Física e Licenciatura e 1 com Educação Física e Bacharelado, conforme as porcentagens apresentadas na Tabela 1.

Tabela1. Quantidade de Publicações por Descritores

\begin{tabular}{lcc}
\hline \multicolumn{1}{c}{ DESCRITORES } & QUANTIDADES & $\%$ \\
\hline Educação Física e Formação Profissional & 25 & 89 \\
Educação Física e Licenciatura & 2 & 7 \\
Educação Física e Bacharelado & 1 & 4 \\
\hline
\end{tabular}

Fonte: Do autor

Não se observa uma linha crescente de publicações nos últimos dez anos e a maior prevalência de publicações encontra-se entre os anos de 2009 e 2012, contendo 21 artigos que tratam desta temática. Vale ressaltar ainda que nos anos de 2005, 2008 e até o mês de Maio/2014 não houve nenhuma publicação. O Gráfico 1 mostra quantitativamente as publicações desta quase uma década.

Gráfico 1. Quantidade de Publicações por Ano.

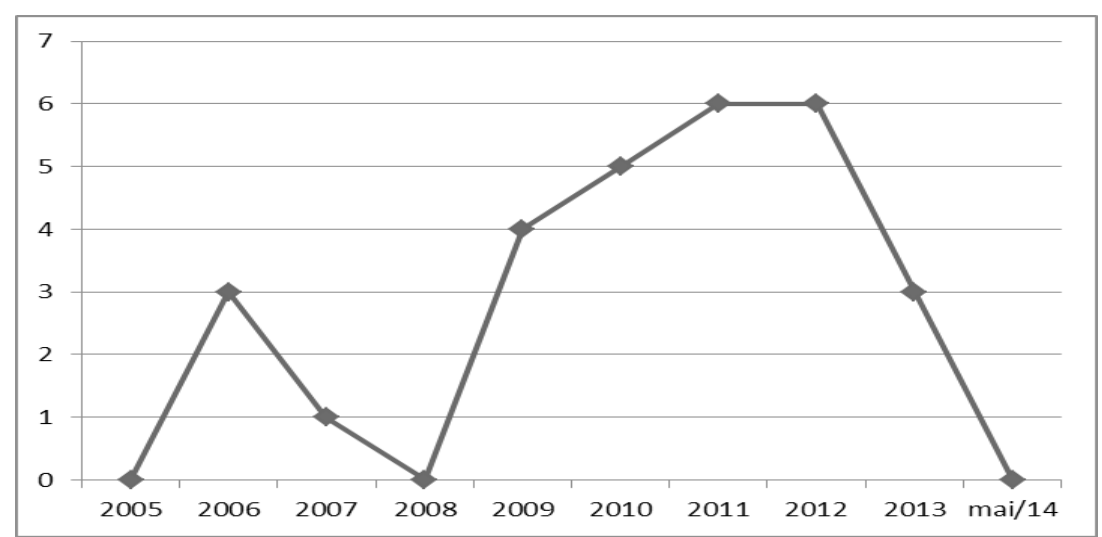

Fonte: Do autor 
Esse dado também pode revelar certa acomodação dos profissionais da área, talvez imaginando que as questões legais devam preceder às análises epistemológicas. Por sinal, a história da EF no Brasil tem dado demonstração do pequeno apreço que os acadêmicos da área têm para produzir conhecimento na tentativa de dotar a EF de pressupostos epistemológicos que possam ajudar a nortear seus profissionais no estabelecimento de uma identidade para a área. Este pode ser um indicativo da baixa produção e da confusão entre as duas habilitações da área, como demonstra o Gráfico 1.

Mais uma hipótese pode ser levantada, mesmo não tendo sido objeto de estudo deste escrito. É senso comum entre docentes e discentes da EF nos cursos superiores, identificarem o Bacharelado como formar profissionais para fora da escola, e a Licenciatura como formar professores. Isto revela não os pressupostos da formação de uma área de conhecimento, e sim a aplicação dos conteúdos encontrados nesses cursos de graduação no mercado de trabalho. Assim, para que produzir estudos sobre formação profissional?

A fim de se ampliar a análise e o entendimento dos resultados desta pesquisa, os artigos encontrados foram divididos por assunto, estabelecendo-se três categorias: 1) Formação/Graduação, 2) Atuação Profissional e 3) Área de Conhecimento, como mostra a tabela 2.

Tabela 2. Quantidade de Publicações por Assunto

\begin{tabular}{ccc}
\hline ASSUNTO & QUANTIDADE & $\%$ \\
\hline Formação/Graduação & 16 & 57 \\
Atuação Profissional & 10 & 36 \\
Área de Conhecimento & 2 & 7 \\
\hline
\end{tabular}

Fonte: Do autor

Enquadraram-se na categoria 1) Formação/Graduação, artigos que tratassem de Projetos Políticos Pedagógicos, de Conteúdos Disciplinares e/ou de Currículo dos cursos de formação em Educação Física (Bacharelado e Licenciatura). Para a categoria 2) Atuação Profissional, foram selecionados artigos que abordassem especificamente os campos de atuação do profissional de Educação Física, sejam eles escolas, academias, clubes, saúde pública, entre outros. E finalmente na categoria 3) Área de Conhecimento, trabalhos que abordassem os objetos de estudo da Área de Conhecimento Educação Física. 
Das publicações encontradas são autores enquadrados na categoria 1) Formação/ Graduação: MUÑOZ et al (2006); BIANCHI, HATJE (2007); ANJOS, DUARTE (2009); BRUGNEROTTO, SIMÕES (2009); KRÜGER, KRUG (2009); FIGUEREDO, HUNGER (2010); LYRA (2010); SILVA (2011); GARANHANI, NADOLNY (2011); BOSSLE, FRAGA (2011); VENTURA (2011); SILVA et al (2012); GÓIS JUNIOR et al (2012); TAVARES, KRUG (2012); NUNES, VOTRE, SANTOS (2012); DE FILIPPIS, MARCELLINO (2013).

$\mathrm{Na}$ categoria 2) Atuação Profissional: COUTINHO, SILVA (2009); COELHO FIILHO, VOTRE (2010); CUNHA et al (2010); GARIGLIO (2010); GOMES et al (2011); FENSTERSEIFER, SILVA (2011); ROSSI, HUNGER (2012); FRAGA, CARVALHO, GOMES (2012); CRUZ JUNIOR, CAPARRÓZ (2013); GARANHANI, MOHAMAD EL TASSA (2013).

Finalmente na categoria 3) Área de Conhecimentos: FÁVARO, et al (2006) e ALVES (2006).

Ao longo deste trabalho mostramos como se deu as publicações em Educação Física quanto à formação de seus profissionais nos últimos aproximadamente 10 anos, assuntos como área de conhecimento e campo de atuação surgiram nos resultados, justificando-se por serem temas codependentes, ou seja, a formação profissional depende da área de conhecimento bem como do campo de atuação.

Nos trabalhos categorizados aqui como sendo de Formação/Graduação, Campo de Atuação e Área de Conhecimento, temas como estruturação de currículos, projetos políticos pedagógicos, conteúdos acadêmicos, ambientes de trabalho e objetos de estudo foram abordados em prol da Educação Física e seus processos legais de formação.

De acordo com Brugnerotto, Simões (2009) ao estudarem a estruturação de currículos de formação com um enfoque em saúde, concluíram que há intencionalidade nas propostas curriculares, porém, se direcionadas para mudanças qualitativas na formação em Educação Física, estas deveriam considerar o ser humano em suas mais diversas manifestações.

Os autores concluem ainda que é necessário repensar as interpretações feitas ao conceito de saúde nos currículos analisados por eles, pois pode haver divergências entre teoria e prática. Este fato deve ser levado à atenção com quaisquer outros conceitos aplicados durante o processo de formação, evitando assim que seja aplicado de forma equivocada. 
A contextualização dos conceitos deve estar garantida no projeto político pedagógico dos cursos de formação, o qual, de acordo com Ventura (2011), não deve ter seus holofotes voltados para as instituições de ensino, seus professores ou sua gestão acadêmica, e sim para o aluno, para o processo de ensino aprendizagem. Deve-se "pensar a relação ensino/ pesquisa/extensão, pensar a relação teoria/prática e pensar a relação interdisciplinar para que a aprendizagem do aluno seja a mais qualificada possível." (VENTURA, p. 87, 2011)

Neste sentido, Silva (2011) objetivando diferenciar o curso de Licenciatura do curso de Bacharelado em Educação Física de uma universidade baiana a partir de seu projeto político pedagógico, aponta um grave problema. Por serem legalmente formações distintas, entende-se que deveriam apresentar também grades curriculares distintas, o que segundo ele não ocorre:

Percebeu-se uma grade curricular muito próxima entre as duas habilitações, com a maioria das disciplinas sendo a mesma em ambas as formações, o que poderia levar a crer em mesmos perfis de professores, mesmos conhecimentos e mesmas práticas. (SILVA, p. 81, 2011).

Seguindo por esta perspectiva, Góis Junior, et al (2012) em um estudo histórico sobre a Escola de Educação Física e Esportes da Universidade de São Paulo (EEFE-USP) relata a influência dos processos de constituição legais da Educação Física no currículo desta instituição. De acordo com estes autores, para a EEFE-USP o esporte e a educação física são fenômenos diferentes, e a partir disso e "levando em consideração o perfil do egresso, optou pela criação de um bacharelado em educação física e um bacharelado em esporte." (GÓIS JUNIOR, et al, p. 399, 2012), tornado a licenciatura um complemento da formação no currículo da Universidade de São Paulo.

Para Krüger, Krug (p. 52, 2009), "ser professor e ser formador de professor associamse às tentativas de mudanças na medida em que estas acompanham o sujeito em seu desenvolvimento profissional”. Seguindo esta concepção, hoje, a área da Educação Física se depara com três realidades de formação profissional, a dos cursos de Licenciatura em Educação Física, a dos cursos de Bacharelado em Educação Física e a dos Cursos de Bacharelado em Esportes, todas, a sua maneira, buscando atender conceitos fracos e confusos a respeito de seus objetos de estudo. 


\section{Considerações Finais}

A partir dos dados apresentados fica evidente um conflito entre os cursos de Formação Profissional em Educação Física, não sendo possível verificar um padrão de formação para diferentes habilitações da área. Nota-se que os próprios estudos que se propõem a discutir essa temática se perdem em seus conceitos, abordando por sua vez questões referentes à área de conhecimento da profissão e seus campos de atuação no mercado de trabalho.

Vale ressaltar que de todos os trabalhos analisados nesta pesquisa poucos trataram da separação entre Licenciatura e Bacharelado em Educação Física, uns dos critérios de inclusão proposto por este estudo, salientando aqui a diminuição gradual das publicações nos últimos anos.

Este estudo abre espaço para novas investigações nesta temática, sejam elas para demarcar como tem se dado na prática a divisão estabelecida em 2002, ou de que maneira os conteúdos da Educação Física e Esportes tem sido ministrado nas diferentes habilitações desta área de conhecimento. 


\section{Referências}

ALVES, Wanderson Ferreira. Sobre a Formação Profissional dos Professores de Educação Física e as Teorias do Saber Docente. Pensar a Prática. v. 9, n. 2, p. 313-330, 2006.

ANJOS, Tatiana Coletto dos; DUARTE, Ana Cláudia Garcia de Oliveira. A Educação Física e a Estratégia de Saúde da Família: formação e atuação profissional. Revista de Saúde Coletiva, Rio de Janeiro, v. 19, n. 4, p. 1127-1144, 2009.

BIANCHI, Paula; HATJE, Marli. A Formação Profissional em Educação Física Permeada pelas Tecnologias de Informação e Comunicação no Centro de Educação Física e Desportos da Universidade Federal de Santa Maria. Revista Pensar a Prática. v. 10, n. 2, 2007.

BOSSLE, Cibele Biehl; FRAGA; Alex Branco. O Personal Trainer na Perspectiva do Marketing. Rev. Bras. Ciênc. Esporte, Florianópolis, v. 33, n. 1, p. 149-162, 2011

BRASIL. Ministério da Educação. Nota Técnica 387/2013/CGLNRS/DPR/SERES/MEC. Brasília, DF, 2013. Disponível em: <http://www.educacao.gov.br/index.php?option=com_c ontent\&viewarticle\&id=18540\&Itemid=1215> Acesso: 18/05/2014.

BRASIL. Secretaria de Educação Superior. Referenciais Curriculares dos Cursos de Bacharelado e Licenciatura. Brasília: Ministério da Educação, 2010.

BRUGNEROTTO, Fábio, SIMÕES,Regina. Caracterização dos currículos de formação profissional em Educação Física: um enfoque sobre saúde. Revista de Saúde Coletiva, Rio de Janeiro, v. 19, n. 1, p. 149-172, 2009.

COELHO FILHO, Carlos Alberto de Andrade; VOTRE, Sebastião Josué. Imagens da prática profissional em academias de ginástica na cidade do rio de janeiro. Rev. Bras. Cienc. Esporte, Campinas, v. 31, n. 3, p. 95-110, 2010.

COUTINHO, Nilton Ferreira; SILVA, Sheila Aparecida Pereira dos Santos. Conhecimento e Aplicação de Métodos de Ensino para os Jogos Esportivos Coletivos na Formação Profissional em Educação Física. Movimento, Porto Alegre, v. 15, n. 01, p. 117-144, 2009. CRUZJUNIOR, Gilson; CAPARRÓZ, Francisco Eduardo. A Juventude Rumo À Docência: Considerações Acerca da Formação Profissional em Educação Física. Rev. Bras. Ciênc. Esporte, Florianópolis, v. 35, n. 1, p. 145-159, 2013. 
CUNHA, Gabriel Barros da; et al. Necessidades de formação para o exercício profissional na perspectiva do treinador de Futebol em função da sua experiência e nível de formação. Motriz, Rio Claro, v.16, n.4, p.931-941, 2010.

DARIDO, Suraya; SILVA, Eduardo. O papel das disciplinas esportivas na formação profissional em Educação Física. In: MOREIRA, Wagner \& SIMÕES, Regina (Orgs). Esporte como Fator de Qualidade de Vida. Piracicaba: Unimep, p. 151-162, 2002.

FÁVARO, Paula Evelise; NASCIMENTO, Glauce Yara do; SORIANO, Jeane Barcelos . O Conteúdo da Intervenção Profissional em Educação Física: O ponto de vista de docentes de um curso de formação profissional. Movimento. Porto Alegre, v.12, n. 02, p. 199-221, 2006.

FENSTERSEIFER, Paulo Evaldo; SILVA, Marlon André Da. Ensaiando O "Novo" Em Educação Física Escolar: A Perspectiva de seus Atores. Rev. Bras. Ciênc. Esporte, Florianópolis, v. 33, n. 1, p. 119-134, 2011.

FERREIRA, Norma Sandra de Almeida. As pesquisas denominadas "Estado da Arte". Educação \& Sociedade, ano XXIII, n. 79, 2002.

FIGUEIREDO, Juliana Frâncica; HUNGER, Dagmar Aparecida Cynthia França. A relevância do conhecimento histórico das ginásticas na formação e atuação do profissional de educação física. Motriz rev. educ. fís. (Impr.), v. 16, n. 1, p. 189-198, 2010.

FILIPPIS, André De; MARCELLINO, Nelson Carvalho. Formação profissional em lazer, nos cursos de Educação Física, no Estado de São Paulo. Movimento. Porto Alegre, v. 19, n. 03, p. 31-56, 2013.

FRAGA, Alex Branco; CARVALHO, Yara Maria de; GOMES, Ivan Marcelo. Políticas de Formação em Educação Física e Saúde Coletiva. Trab. Educ. Saúde, Rio de Janeiro, v. 10 n. 3, p. 367-386, 2012.

GARANHANI, Marynelma Camargo; NADOLNY, Lorena de Fátima. A educação física na educação escolar da criança pequena: uma proposta de formação e desenvolvimento profissional de professores. Nuances: estudos sobre Educação. v. 18, n. 19, p. 27-35, 2011.

GARANHANI, Marynelma Camargo; MOHAMAD EL TASSA, Khaled Omar. Formação profissional para atuação em projetos sociais: no foco a formação de professores no 
"programa Segundo Tempo". Movimento. Porto Alegre, v. 19, n. 04, p. 273-287, 2013.

GARIGLIO, José Ângelo. O Papel da Formação Inicial no Processo de Constituição da Identidade Profissional de Professores de Educação Física. Rev. Bras. Ciênc. Esporte, Florianópolis, v. 32, n. 2-4, p. 11-28, 2010.

GÓIS JUNIOR, Edivaldo; et al. Estudo histórico sobre a formação profissional na Escola de Educação Física e Esporte da Universidade de São Paulo (1980-1990). Motriz, Rio Claro, v.18, n.2, p.393-400, 2012.

GOMES, Rúben Emanuel; ISIDRO, Ana Sofia Marques, BATISTA, Paula Fazendeiro; MESQUITA, Isabel Ribeiro. Acesso à carreira de treinador e reconhecimento das entidades responsáveis pela formação: um estudo com treinadores portugueses em função do nível da escolaridade e da experiência profissional. Revista da Educação Física/UEM. Maringá, v.22, n. 2, 2011.

KRÜGER, Leonardo Germano. KRUG, Hugo Norberto. Licenciatura em Educação Física: Concepções a partir da Vivência Experienciada dos Professores do Ensino Superior em seu Percurso Formativo. Movimento, Porto Alegre, v. 15, n. 01, p. 51-70, 2009.

LYRA, Vanessa Bellani. Nos rastros da formação profissional: o projeto de legitimação da Escola Superior de Educação Física de Florianópolis a partir do currículo oficial. Movimento, Porto Alegre, v. 16, n. 01, p. 169-190, 2010.

MUÑOZ, Gabriel Humberto; et al. Reforma Curricular dos Cursos de Licenciatura em Educação Física: Contribuições dos Professores das Redes Públicas de Ensino. Pensar a Prática, v. 9, n.2. p. 231-248, 2006.

NUNES, Marcello Pereira; VOTRE, Sebastião Josué; SANTOS, Wagner dos. O profissional em educação física no Brasil: Desafios e perspectivas no mundo do trabalho. Motriz, Rio Claro, v.18 n.2, p.280-290, 2012.

RANGEL-BETTI, Irene; BETTI, Mauro. Novas Perspectivas na Formação Profissional em Educação Física. Revista Motriz, v. 02, n. 01, jun. 1996.

ROSSI, Fernanda; HUNGER, Dagmar. As etapas da carreira docente e o processo de formação continuada de professores de Educação Física. Rev. bras. Educ. Fís. Esporte, São Paulo, v.26, n.2, p.323-38, 2012. 
SILVA, Osni Oliveira Noberto da. Licenciatura e Bacharelado em Educação Física: diferenças e semelhanças. Revista Espaço Acadêmico. n. 124, 2011.

SILVA, Sheila Aparecida Pereira dos Santos; et al. Atividades acadêmico-científicoculturais na formação do profissional de Educação Física. Motriz, Rio Claro, v.18 n.1, p.92103, 2012.

TAVARES, Julio Emerson Baumart; KRUG, Hugo Norberto. Formação do profissional em educação física: um olhar para o processo de inclusão e inserção social. Revista do Centro de Educação. v. 2003, n. 21, 2012.

TEREZANI, O. L. A avaliação discente nas disciplinas práticas no curso de graduação em Educação Física na Unimep: um estudo de caso. 1994. Dissertação (Mestrado em Educação) - Faculdade de Educação, Universidade Metodista de Piracicaba.

VENTURA, Paulo Roberto Veloso. Universidade: espaço privilegiado para a formação de professores de Educação Física. Linhas Críticas, Brasília, DF, v. 17, n. 32, p. 77-96, 2011. 


\section{VOCATIONAL TRAINING IN PHYSICAL EDUCATION: STATE OF THE ART}

ABSTRACT: The objective of this study is to analyze how the literary production in Physical Education (EF) and Sports is after separation the Bachelor and Degree. Methodology: A review study, the type state of the art, which qualitatively analyzed the literary production in EF. Results: 28 articles were found and divided by subject, establishing three categories: 1) Training / Graduate, 16 articles, 2) Professional Performance, 10 articles, 3) Knowledge Area, 2 articles. Conclusion: It was evidently a conflict between courses of Vocational Training in EF, it is not possible to verify a standard of training for different skills area.

Keywords: Physical Education and Training. Sports.

\section{FORMACIÓN EN EDUCACIÓN FÍSICA: ESTADO DEL ARTE}

RESUMEN: El objetivo de este estudio es analizar cómo es la producción literaria en Educación Física (EF) y Deportes después de la separación entre Licenciatura y Bachelor. Metodología: Estudio de revisión, el tipo estado del arte, que analizó cualitativamente la producción literaria en EF. Resultados: Se encontraron 28 artículos y divididas por temas, estableciendo tres categorías: 1) Formación / graduado, 16 artículos, 2) Práctica Profesional, 10 artículos, 3) Área de Conocimiento, 2 artículos. Conclusión: Era evidentemente un conflicto entre los cursos de Formación Profesional en EF, no es posible verificar un estándar de formación para el área de habilidades diferentes.

Palabras clave: Educación y Entrenamiento Físico. Desportes. 